\title{
The Gamma-Ray Burst Monitor for Lobster-ISS $\left(^{*}\right)$
}

F. Frontera $\left({ }^{1}\right)\left({ }^{2}\right)$, L. Amati $\left({ }^{2}\right)$, N. Auricchio $\left({ }^{2}\right)$, E. Caroli $\left({ }^{2}\right)$, A. Basili $\left({ }^{2}\right)$

A. Bogliolo $\left({ }^{3}\right)$, G. Di Domenico $\left({ }^{1}\right)$, T. Franceschini $\left({ }^{2}\right)$, C. Guidorzi $\left({ }^{4}\right)$

G. Landini $\left({ }^{2}\right)$, N. Masetti $\left({ }^{2}\right)$, E. Montanari $\left({ }^{1}\right)$, M. Orlandini $\left({ }^{2}\right)$

E. Palazzi $\left({ }^{2}\right)$, S. Silvestri $\left({ }^{2}\right)$, J. B. Stephen $\left({ }^{2}\right)$ and G. Ventura $\left({ }^{2}\right)$

$\left({ }^{1}\right)$ Università di Ferrara - Via Saragat 1, Ferrara, Italy

$\left(^{2}\right)$ INAF-Istituto di Astrofisica Spaziale e Fisica cosmica - via P. Gobetti 101, Bologna, Italy

$\left.{ }^{3}\right)$ STI, Universitá di Urbino - Piazza della Repubblica, 13, Urbino, Italy

$\left(^{4}\right)$ Liverpool John Moores University - Liverpool, UK

(ricevuto il 23 Maggio 2005; pubblicato online il 28 Ottobre 2005)

Summary. - We describe the Gamma Ray Burst Monitor (GRBM) that flanks the $\mathrm{X}$-ray telescope for the Lobster-ISS mission.

PACS 98.70.Rz - $\gamma$-ray sources; $\gamma$-ray bursts.

PACS 01.30.Cc - Conference proceedings.

\section{1. - Introduction}

Lobster-ISS [1] is an X-ray mission proposed by an International collaboration led by George Fraser (University of Leicester, UK) in response to the ESA call for two fleximissions (F2 and F3) for the International Space Station (ISS). The project ha already passed an industrial phase-A study funded by ESA for its accommodation aboard the Columbus External Payload Facility for a launch in 2009 and a 3 year duration flight. Unfortunately, given the new NASA policy about ISS, the prospects of a Lobster-ISS flight aboard Columbus are uncertain. Thus other flight opportunities, like that of using another payload facility aboard ISS or a free-flyer satellite, are being investigated.

The main scientific objective of Lobster-ISS is the mapping of the X-ray sky in the 0.1-3.5 keV energy band with an angular resolution as low as 4-6 arcmin and a daily sensitivity of $2 \times 10^{-12} \mathrm{erg} \mathrm{cm}^{-2} \mathrm{~s}^{-1}$. The main instrument is based on Micro-Channel Plate (MCP) optics in a Lobster-eye configuration and focal plane detectors based on special sensitive proportional counters. It is composed of six identical modules, each with a Field of View (FOV) of $27^{\circ} \times 22.5^{\circ}$, misaligned in such a way to give a total rectangular field of view of $22.5^{\circ}$ in the direction of the ISS motion and $162^{\circ}$ in the perpendicular direction. Thanks to the orbital motion of the ISS, it will be possible to map almost all the sky every orbit, allowing the production of a catalog of $250000 \mathrm{X}$-ray

(*) Paper presented at the "4th Workshop on Gamma-Ray Burst in the Afterglow Era", Rome, October 18-22, 2004.

(C) Società Italiana di Fisica 


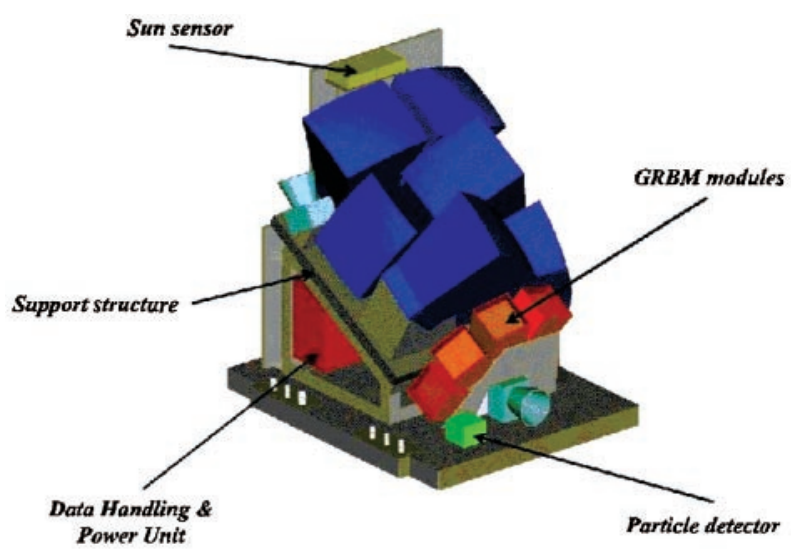

Fig. 1. - Sketch of the Lobster payload. The location of the GRBM modules is indicated (courtesy of Carlo Gavazzi Space).

sources every two months. The combination of the wide field of view, the good angular resolution and the high sensitivity will allow the study of the time behaviour of all classes of X-ray sources, inclusive of Gamma-Ray Bursts (GRBs). Given that many classes of sources (e.g., flare stars, compact X-ray binaries) are emitters of short transient events, the separation of GRBs from other X-ray short events is a hard task for an instrument with a passband from 0.1 to $3.5 \mathrm{keV}$. In order to overcome this issue, a Gamma Ray Burst Monitor (GRBM) flanks the Lobster-eye telescope. In this paper we describe the GRBM proposed for the phase-A study, its science goals and performance.

\section{2. - The Lobster-ISS GRBM}

The GRBM proposed consists of 4 misaligned detection units, each one made of an array of Peltier-cooled CdZnTe (CZT) detectors, surmounted by a passive collimator which defines the field of view. The unit FOV $35^{\circ} \times 55^{\circ}(\mathrm{FWHM})$, resulting in a total rectangular field of view of $35^{\circ}$ in the direction of the ISS motion and $240^{\circ}$ in the perpendicular direction. Each collimator is made of four slabs of $1.5 \mathrm{~mm}$ thick Tungsten with a graded shield to minimize the X-ray fluorescence. With this instrument configuration, a source is viewed by at least 2 detection units for most $(95 \%)$ of the directions within the FOV of Lobster. As a consequence, the area exposed by the GRBM to these directions ranges from 0.70 to 1.30 times the useful area of a single detection unit.

A sketch of the Lobster-ISS payload configuration is shown in fig. 1. Each detection unit is made of an array of CZT elementary crystals (pixels) [2]. Each pixel has a cross section of $8 \times 8 \mathrm{~mm}^{2}$. The elementary crystals are packed together in sub-units of $4 \times 4$ pixels, while each detection unit is made of $3 \times 6$ sub-units. Thus the X-ray sensitive array is made of 288 pixels. The active area of each unit is $184 \mathrm{~cm}^{2}$ while its geometric area, which takes into account a $0.5 \mathrm{~mm}$ pitch between each couple of pixels, is $208 \mathrm{~cm}^{2}$. The crystals are assembled on thin $(1 \mathrm{~mm})$ ceramic plates. Below each module is located the front-end electronics with multiplexers and ADCs. The detector thickness, which was assumed for the phase A study in order to guarantee an instrument passband from 3 to $300 \mathrm{keV}$, is $5 \mathrm{~mm}$. We are evaluating the extension of the energy passband up to $700 \mathrm{keV}$ either by increasing the CZT thickness up to $10 \mathrm{~mm}$ or by using an alternative 


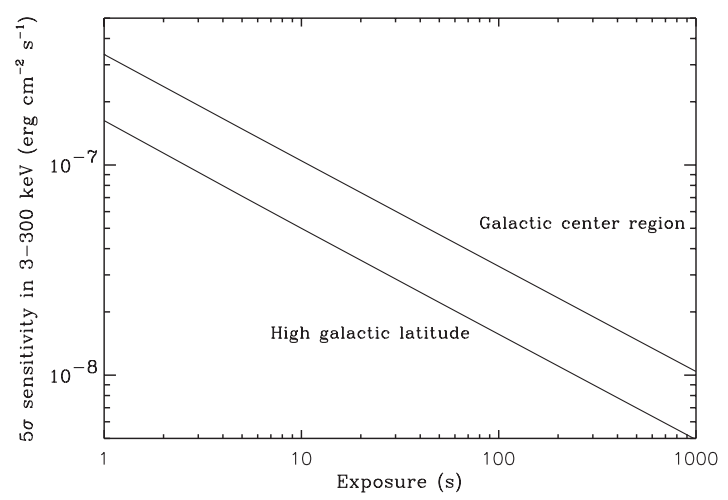

Fig. 2. - Expected $5 \sigma$ sensitivity of the GRBM to a GRB with typical spectrum (see text) as a function of the exposure time. Two cases have been considered: a source located in the direction of the galactic center region and source at high galactic latitude.

detector, like a phoswich-like detector made of a silicon drift chamber coupled with a CsI scintillator, the development of which is underway [3]. In the case of a GRB detection, the GRBM electronics and on-board data handling provide high-resolution spectra and light curves. The GRB position is also determined on board and automatically transmitted to the Lobster data handling electronics for a better position determination by means of the X-ray telescope.

\section{3. - Expected performance}

The mean background level at the ISS orbit has been estimated by summing the contributions of the diffuse X-ray background and the intrinsic (particle-induced) background. Given the wide field of view of the GRBM, the contribution of bright galactic sources to the total background level is not negligible. In particular, when the Galactic Bulge is inside the FOV of a detection unit, the background level is expected to increase by about $30 \%$. This has been taken into account in the performance estimates and numerical simulations.

The $5 \sigma$ sensitivity of the GRBM to a GRB source in the $3-300 \mathrm{keV}$ energy band is shown in fig. 2 as a function of the time exposure, either in the case of a source located in the direction of the galactic center region or for a source at high galactic latitude. For the sensitivity evaluation we have assumed as spectral model a typical Band function [4] with $\alpha=-1, \beta=-2$ and $E_{0}=200 \mathrm{keV}$, Conservatively, for the area exposed to the source, we have assumed that corresponding to the minimum $\left(129 \mathrm{~cm}^{2}\right)$, while as instrumental background level we have assumed that corresponding to an area of $2 \times 184=368 \mathrm{~cm}^{2}$. We note that, by means of an off-line refined analysis, we expect to be able to identify the pixels (or groups of pixels) illuminated by the source. In this case the detection area contributing to the background level will be reduced to that exposed to the source and the instrument sensitivity will be significantly improved with respect to the values reported in fig. 2. The expected source localization accuracy at $90 \%$ confidence level as a function of the burst fluence in the $50-300 \mathrm{keV}$ energy band ranges from $\sim 1^{\circ}-2^{\circ}$ for the brightest events $\left(\geq 5 \times 10^{-5} \mathrm{erg} \mathrm{cm}^{-2}\right.$ for a $20 \mathrm{~s}$ burst $)$ to several tens of degrees for the weakest ones $\left(\sim 5 \times 10^{-7} \mathrm{erg} \mathrm{cm}^{-2}\right.$ for a $20 \mathrm{~s}$ burst $)$. For the location accuracy we have assumed the above Band function, and as detection area that illuminated by the GRB. We used 
the source localization reconstruction algorithm similar to that adopted to localize the GRB, detected with the BeppoSAX GRBM [5]. One of the main goals of the present GRBM configuration is the possibility of detecting transient absorption features during the rise of the GRB events. Such features, possibly associated with a variable column density, are predicted by several models and have already been observed in two cases (GRB990705 [6]; GRB011211 [7]). The joint fitting of the GRBM and Lobster spectra will extend the analysis down to $0.1 \mathrm{keV}$, allowing not only a better study of the column density behaviour with time, but also a higher significance of the absorption features. In the case of the $13 \mathrm{~s}$ transient feature from GRB990705, from numerical simulations we have found that the GRBM for Lobster-ISS will be capable to detect the feature at $3.8 \mathrm{keV}$ at a much higher statistical significance than that achieved with BeppoSAX [6]. With the present GRBM configuration, we also expect to be capable of measuring the polarization of gamma-ray photons [8]. For a GRB with $25-100 \mathrm{keV}$ fluence similar to that of GRB $021206\left(2.9 \times 10^{-5} \mathrm{erg} \mathrm{cm}^{-2}[9]\right)$, the expected minimum detectable linear polarization is $\sim 60 \%$ in the $70-150 \mathrm{keV}$ energy range and $\sim 30 \%$ in the $150-300 \mathrm{keV}$ band.

\section{4. - Conclusions}

Thanks to the GRBM, the Lobster-ISS passband extends from 0.1 to $300 / 700 \mathrm{keV}$, an unprecedented energy band never used by an all-sky monitor to study the prompt emission of GRBs. The very good energy resolution of the GRBM, $3 \%$ at $6 \mathrm{keV}$, will permit a sensitive study of transient absorption and/or emission features during the early phase of the prompt emission. Moreover, the combination of the two instruments will allow an unprecedented study of the absorption cut-offs below $2 \mathrm{keV}$ in the GRB spectra. The study of these features and cut-offs is of key importance for the determination of the circumburst environment properties, the nature of progenitors and the connection with SNe. The proposed GRBM can also be exploited to measure the polarization of the GRB prompt emission. In the described configuration, Lobster-ISS is also the ideal mission for studying X-Ray Flashes (XRFs) and their nature. In addition to GRBs and XRFs, the GRBM will allow identification and study of all types of fast high energy X-ray transients, in particular of the Soft Gamma-Ray Repeaters (e.g., [10]). A more detailed description of the instrument scientific case, design and expected performances can be found in [11].

\section{REFERENCES}

[1] Fraser G., Brunton A.N., Bannister N.P. et al., Proc. SPIE, 4497 (2002) 115.

[2] Caroli E., Stephen J.B., Auricchio N. et al., Proc. SPIE, 3765 (1999) 597.

[3] Marisaldi M., Labanti C., Bulgarelli A. et al., NewA, 48 (2004) 305.

[4] Band D., Matteson J., Ford L. et al., ApJ., 413 (1993) 281.

[5] Guidorzi C., Frontera F., Montanari E. et al., Exploring the gamma-ray universe, in Proceedings of the Fourth INTEGRAL Workshop, edited by Gimenez A., Reglero V. and Winkler C. (ESA Publications Division, Noordwijk) 1997, pp. 449-452.

[6] Amati L., Frontera F., Vietri M. et al., Science, 290 (2000) 953.

[7] Frontera F., Amati L., in 't Zand J. J. M. et al., ApJ, 616 (2004) 1078.

[8] Curado da Silva R. M., Auricchio N., Caroli E. et al., IEEE Transactions on Nuclear Science, 51 (2004) 2478.

[9] Coburn W. and Boggs S. E., Nature, 423 (2003) 415.

[10] Guidorzi C., Frontera F., Montanari E. et al., A $\& A$, 416 (2004) 297.

[11] Amati L., Frontera F., Auricchio N. et al., Adv. Sp. Res., in press (2005). 